\title{
On complementable operators in the sense of T. Ando
}

\author{
M. Laura Arias ${ }^{1,2, a}$, Gustavo Corach $^{1,2, b}$ and Alejandra Maestripieri ${ }^{1,2, c *}$
}

AMS Classification: 47A64, 47B65

Keywords: Schur complements, shorted operators, Wiener-Hopf operators, minus order

\begin{abstract}
Consider an operator $A: \mathcal{H} \rightarrow \mathcal{K}$ between Hilbert spaces and closed subspaces $\mathcal{S} \subset \mathcal{H}$ and $\mathcal{T} \subset \mathcal{K}$. If there exist projections $E$ on $\mathcal{H}$ and $F$ on $\mathcal{K}$ such that $R(E)=\mathcal{S}, R(F)=\mathcal{T}$ and $A E=F^{*} A$ then $A$ is called $(\mathcal{S}, \mathcal{T})$-complementable. The origin of this notion comes from the idea of T. Ando of defining Schur complements in terms of operators. In this paper we present some characterizations of these triples $(A, \mathcal{S}, \mathcal{T})$ and applications to bilateral Schur complements and generalized Wiener-Hopf operators.
\end{abstract}

\section{Introduction}

Given a square block matrix

$$
A=\left(\begin{array}{ll}
B & C \\
D & E
\end{array}\right)
$$

where $B$ and $E$ are square and $B$ is invertible, the Schur complement of $B$ in $A$ is $E-D B^{-1} C$. The name, due to E. Haynsworth [20], comes from the formula $\operatorname{det} A=\operatorname{det} B \operatorname{det}\left(E-D B^{-1} C\right)$, discovered by I. Schur [30]. By changing the inversion by generalized inverses, the notion is available also for non-square block matrices and milder invertibility hypothesis. On the other side, there exists an extensive theory on the so-called shorted operators. M. G. Krein [23], in his studies about selfadjoint extensions of some operators on a Hilbert space defined, for a positive semidefinite operator $A$ on $\mathcal{H}$ and a closed subspace $\mathcal{S}$ of $\mathcal{H}$, the shorted operator $A_{/ \mathcal{S}}$ by means of the existence of the maximum of $\{B \in L(\mathcal{H}): 0 \leq B \leq A, R(B) \subseteq \mathcal{S}\}$. See also Pekarev-Šmuljan [28] and Arlinskii and E. Tsekanovskii [5]. This notion was rediscovered by Anderson and Trapp [2], who, among many other results, proved that $A_{/ \mathcal{S}}$ coincides with the Schur complement. It is impossible to record the different situations, disciplines and applications which rely on these "complements". We refer the reader to the excellent surveys by R. W. Cottle [13] and D. Carlson [8] and the more recent book edited by F. Zhang [33]. We do mention a note by C. A. Butler and T. D. Morley [7] which compares six different extensions and generalizations of Schur complements. One among them, due to T. Ando [3] is the origin of this note. Given an $n \times n$ complex matrix $A$ and a subspace $\mathcal{S}$ of $\mathbb{C}^{n}, A$ is called $\mathcal{S}$-complementable if there exist matrices $M_{r}, M_{l}$ such that $P_{\mathcal{S}} M_{r}=M_{r}, M_{l} P_{\mathcal{S}}=M_{l}, P_{\mathcal{S}} A M_{r}=P_{\mathcal{S}} A$ and $M_{l} A P_{\mathcal{S}}=A P_{\mathcal{S}}$; here $P_{\mathcal{S}}$ denotes the orthogonal projection onto $\mathcal{S}$. The matrices $A_{\mathcal{S}}:=A M_{r}$ and $A_{\mathcal{S}}:=A-A_{\mathcal{S}}$ are called the Schur compression of $A$ to $\mathcal{S}$ and the generalized Schur complement of $A$ with respect to $\mathcal{S}$. Ando proved that if

${ }^{*}$ M. L. Arias was partially supported by CONICET (PIP 11220120100426), FONCYT (PICT 2017-0883 and 2014-1776), G. Corach by CONICET (PIP 11220120100426), FONCYT (PICT 2014-1776) and A. Maestripieri by CONICET (PIP 11220130100168) 
$A=\left(\begin{array}{ll}B & C \\ D & E\end{array}\right), \mathbb{C}^{n}=\mathbb{C}^{n_{1}} \oplus \mathbb{C}^{n_{2}}$ and the $n_{1} \times n_{1}$ matrix $B$ is invertible then $M_{r}=\left(\begin{array}{cc}I & B^{-1} C \\ 0 & 0\end{array}\right)$, and $M_{l}=\left(\begin{array}{cc}I & 0 \\ D B^{-1} & 0\end{array}\right)$, show that $A$ is $\mathbb{C}^{n_{1}} \times\{0\}$-complementable and $A_{\mathcal{S}}=\left(\begin{array}{cc}B & C \\ D & D B^{-1} C\end{array}\right), A_{/ \mathcal{S}}=$ $\left(\begin{array}{cc}0 & 0 \\ 0 & E-D B^{-1} C\end{array}\right),\left(\right.$ here $\mathcal{S}=\mathbb{C}^{n_{1}} \times\{0\}$, of course). Ando's definition have many advantages. We only mention two. Firstly, it has a neat extension to Hilbert space operators (see [11]); secondly, it can be easily extended, at least for matrices, to linear transformations between different vector spaces (i.e., non square matrices) with a fixed subspace on each. This extension is due to Mitra and Puri [26] and, independently, to Carlson and Haynsworth [9]. Much later, Antezana et al. [4] extended this idea to Hilbert space operators: for a bounded linear operator $A: \mathcal{H} \rightarrow \mathcal{K}$ between Hilbert spaces and closed subspaces $\mathcal{S} \subset \mathcal{H}, \mathcal{T} \subset \mathcal{K}$, they gave appropriate definitions of complementability and a weaker notion called weak complementability which allows the definition of a generalized bi-Schur complement (or, bilateral shorted operator) $A_{/ \mathcal{S}, \mathcal{T}}$. Antezana et al. [4] applied this construction to the extension of parallel sums of bounded linear operators. This study started with Anderson and Duffin [1]; see also Filmore-Williams [18]. The present paper continues their research in different directions. On the one hand, we explore in more detail several features of the complementability. On the other hand, we use them to get results on the operators $T_{\mathcal{T}, \mathcal{S}}(A)=\left.P_{\mathcal{T}} A\right|_{\mathcal{S}}: \mathcal{S} \rightarrow \mathcal{T}$ which Devinatz and Shinbrot [15] called general Wiener-Hopf operators.

It should be mentioned that the theory of compatibility between subspaces and selfadjoint operators plays a significant role in this paper, mainly through its methods. This theory, first introduced by Hassi and Nordström [19], was later developed in a large series of papers (among them, [10], [11], [12]). It should be noticed that a selfadjoint operator $A$ and a closed subspace $\mathcal{S}$ are compatible if and only if $A$ is $\mathcal{S}$-complementable (see [11]).

We briefly describe the contents of the paper. In Section 2 we study complementable and weakly complementable operators. The main results of this section are Propositions 2.4 and 2.15, where we prove that the notion of complementable operators (resp. weakly complementable operators) introduced by Antezana et al. in [4] is equivalent to the existence of certain bounded projections (resp. densely defined projections). In addition, we fully describe those projections. In Section 3 we study the generalized invertiblity (or, invertiblity) of general Wiener-Hopf operators through the notions of complementable and weakly complementable operators. For instance, under the condition of weakly complementability, we obtain a formula of the Moore-Penrose inverse of this kind of operators by means of the projections associated to this condition. The main contributions of this section are Theorems 3.2, 3.6 and 3.7. Finally, in Section 4 we study bilateral shorted operators. As we mentioned before, this class of operators was introduced in [4] under the hypothesis of weakly complementability. Here, we present a formula of this operator by means of the projections associated to the weakly complementability, see Theorem 4.2. Moreover, in Theorem 4.6 we provide a variational characterization of the bilateral shorted operator which extends a result given in [4].

\section{Complementable and weakly-complementable operators}

Throughout this paper $\mathcal{G}, \mathcal{H}, \mathcal{K}$ denote complex Hilbert spaces. $L(\mathcal{H}, \mathcal{K})$ is the space of all bounded linear operators from $\mathcal{H}$ to $\mathcal{K}$ and the algebra $L(\mathcal{H}, \mathcal{H})$ is abbreviated by $L(\mathcal{H})$. Given $T \in L(\mathcal{H}, \mathcal{K})$ its range is denoted by $R(T)$, its nullspace by $N(T)$ and its adjoint by $T^{*}$. We denote by $T^{\dagger}$ the Moore-Penrose inverse of $T$. Along this article we shall deal with not necessarily bounded projections. A densely defined operator $E$, with domain $\mathcal{D}(E)$, is a called projection if $R(E) \subseteq \mathcal{D}(E)$ and $E(E x)=E x$ for all $x \in \mathcal{D}(E)$. In this case, $\mathcal{D}(E)=R(E)+N(E)$, see [27]. The set of bounded (oblique) projections in $L(\mathcal{H})$ is denoted by $\mathcal{Q}(\mathcal{H})$ and the set of orthogonal projections in $L(\mathcal{H})$ by 
$\mathcal{P}(\mathcal{H})$, i.e., $\mathcal{Q}(\mathcal{H})=\left\{E \in L(\mathcal{H}): E^{2}=E\right\}$ and $\mathcal{P}(\mathcal{H})=\left\{P \in \mathcal{Q}(\mathcal{H}): P=P^{*}\right\}$. To simplify the notation, we will often omit the Hilbert space and just write $\mathcal{Q}, \mathcal{P}$ when no confusion arises. For two fixed closed subspace $\mathcal{S}, \mathcal{T}$ of $\mathcal{H}$ such that $\mathcal{H}=\mathcal{S}+\mathcal{T}$, denote by $Q_{\mathcal{S} / / \mathcal{T}}$ the element in $\mathcal{Q}$ with $R\left(Q_{\mathcal{S} / / \mathcal{T}}\right)=\mathcal{S}$ and $N\left(Q_{\mathcal{S} / / \mathcal{T}}\right)=\mathcal{T}$ and by $P_{\mathcal{S}}$ the element in $\mathcal{P}$ with $R\left(P_{\mathcal{S}}\right)=\mathcal{S}$. The next result will be frequently applied along this note, see [14, Theorem 22] for its proof.

Theorem 2.1. If $A, B \in L(\mathcal{H})$ have closed ranges, then $A B$ has closed range if and only if $R(B)+$ $N(A)$ is closed.

Along this article, $A \in L(\mathcal{H}, \mathcal{K})$ and $\mathcal{S} \subseteq \mathcal{H}$ and $\mathcal{T} \subseteq \mathcal{K}$ are closed subspaces.

\subsection{Complementability}

In what follows we study complementable operators. This notion was introduced by T. Ando [3] in finite dimensional spaces with the aim of studying the Schur compression and Schur complement of matrices. Later this concept was generalized for two subspaces by Mitra and Puri [26] and Carlson and Haynsworth in [9], and extended to infinite dimensional Hilbert spaces by Antezana et al. in [4]. Although our next definition of complementability differs from the one considered in [4], we show in Proposition 2.4 that both definitions coincide.

Definition 2.2. An operator $A \in L(\mathcal{H}, \mathcal{K})$ is $(\mathcal{S}, \mathcal{T})$-complementable if there exist two projections $E \in \mathcal{Q}(\mathcal{H}), F \in \mathcal{Q}(\mathcal{K})$ with $R(E)=\mathcal{S}, R(F)=\mathcal{T}$ such that $A E=F^{*} A$.

In other words, $A \in L(\mathcal{H}, \mathcal{K})$ is $(\mathcal{S}, \mathcal{T})$-complementable if there exist two bounded projections $E, F$ onto $\mathcal{S}, \mathcal{T}$ respectively, such that $F$ is an $A$-adjoint of $E$, i.e., $\langle E x, y\rangle_{A}=\langle x, F y\rangle_{A}$, for all $x \in \mathcal{H}, y \in \mathcal{K}$ where $\langle x, y\rangle_{A}:=\langle A x, y\rangle$. When $\mathcal{H}=\mathcal{K}$ and $\mathcal{S}=\mathcal{T}$ we say that $A \in L(\mathcal{H})$ is $\mathcal{S}$-complementable when $A$ is $(\mathcal{S}, \mathcal{S})$-complementable. We denote by $\mathcal{P}(A, \mathcal{S}, \mathcal{T})$ the set of projections arising from 2.2 , i.e.,

$$
\mathcal{P}(A, \mathcal{S}, \mathcal{T}):=\left\{(E, F) \in \mathcal{Q} \times \mathcal{Q}: R(E)=\mathcal{S}, R(F)=\mathcal{T}, A E=F^{*} A\right\} .
$$

Clearly, the set $\mathcal{P}(A, \mathcal{S}, \mathcal{T})$ is not empty if and only if $A$ is $(\mathcal{S}, \mathcal{T})$-complementable.

Remarks 2.3. The next properties are straightforward:

1. $A$ is $(\mathcal{S}, \mathcal{T})$-complementable if and only if $A^{*}$ is $(\mathcal{T}, \mathcal{S})$-complementable. In this case, $(E, F) \in \mathcal{P}(A, \mathcal{S}, \mathcal{T})$ if and only if $(F, E) \in \mathcal{P}\left(A^{*}, \mathcal{T}, \mathcal{S}\right)$

2. A selfadjoint operator $A \in L(\mathcal{H})$ is $\mathcal{S}$-complementable if and only if there exists a projection $E$ with $R(E)=\mathcal{S}$ such that $A E=E^{*} A$. In fact, if $A$ is $\mathcal{S}$-complementable, there exist two projections $E, F \in \mathcal{Q}$ with $R(E)=R(F)=\mathcal{S}$ such that $A E=F^{*} A$ then $E^{*} A E=E^{*} F^{*} A=$ $E^{*} A$ is selfadjoint and so $A E=E^{*} A$ as desired. The converse is straightforward. Therefore, if $A$ is a selfadjoint operator then $A$ is $\mathcal{S}$-complementable if and only if $(A, \mathcal{S})$ is compatible in the sense of [10].

Let us start by proving that our definition of $(\mathcal{S}, \mathcal{T})$-complementability is equivalent to the definition given by Carlson and Haynsworth in [9].

Proposition 2.4. The following conditions are equivalent:

1. A is $(\mathcal{S}, \mathcal{T})$-complementable. 
2. Given $Q_{s} \in \mathcal{Q}(\mathcal{H})$ and $Q_{t} \in \mathcal{Q}(\mathcal{K})$ with $R\left(Q_{s}\right)=\mathcal{S}$ and $N\left(Q_{t}\right)=\mathcal{T}^{\perp}$ there exist operators $M_{r} \in L(\mathcal{H}), M_{l} \in L(\mathcal{K})$ such that $M_{l} Q_{t}=M_{l}, Q_{s} M_{r}=M_{r}, M_{l} A Q_{s}=A Q_{s}$ and $Q_{t} A M_{r}=$ $Q_{t} A$.

Proof. $1 \Rightarrow 2$. Assume that $A$ is $(\mathcal{S}, \mathcal{T})$-complementable and let $E, F$ be two projections with $R(E)=\mathcal{S}$ and $R(F)=\mathcal{T}$ such that $A E=F^{*} A$. Consider two projections $Q_{s}, Q_{t}$ with $R\left(Q_{s}\right)=\mathcal{S}$ and $N\left(Q_{t}\right)=\mathcal{T}^{\perp}$. Then, $F^{*} Q_{t}=F^{*}, Q_{s} E=E, F^{*} A Q_{s}=A E Q_{s}=A Q_{s}$ and $Q_{t} A E=Q_{t} F^{*} A=$ $Q_{t} A$. Hence, item 2 holds, with $M_{r}=E$ and $M_{l}=F^{*}$.

$2 \Rightarrow 1$. Let $Q_{s}, Q_{t}$ be two projections with $R\left(Q_{s}\right)=\mathcal{S}$ and $N\left(Q_{t}\right)=\mathcal{T}^{\perp}$ and $M_{r}, M_{l}$ be two operators such that $M_{l} Q_{t}=M_{l}, Q_{s} M_{r}=M_{r}, M_{l} A Q_{s}=A Q_{s}$ and $Q_{t} A M_{r}=Q_{t} A$. Define $E:=$ $Q_{s}+M_{r}\left(I-Q_{s}\right)$ and $F:=Q_{t}^{*}+M_{l}^{*}\left(I-Q_{t}\right)^{*}$. Thus, from $Q_{s} M_{r}=M_{r}$ and $M_{l} Q_{t}=M_{l}$, we obtain that $E^{2}=E, F^{2}=F$ and $R(E)=R\left(Q_{s}\right)=\mathcal{S}$ and $R(F)=R\left(Q_{t}^{*}\right)=\mathcal{T}$. We claim that $A E=F^{*} A$. In fact, $F^{*} A E=\left(Q_{t}+\left(I-Q_{t}\right) M_{l}\right) A E=Q_{t} A E+\left(I-Q_{t}\right) M_{l} A Q_{s} E=Q_{t} A E+\left(I-Q_{t}\right) A E=A E$, where we use that $M_{l} A Q_{s}=A Q_{s}$ and $Q_{s} E=E$. In a similar way $F^{*} A E=F^{*} A$.

In the next result we present equivalent conditions to the notion of complementability in terms of ranges of operators and Hilbert spaces decompositions. In the sequel, we will frequently use that $(A \mathcal{S})^{\perp}=\left(A^{*}\right)^{-1}\left(\mathcal{S}^{\perp}\right)$.

Proposition 2.5. The following conditions are equivalent:

1. A is $(\mathcal{S}, \mathcal{T})$-complementable.

2. $R\left(P_{\mathcal{S}} A^{*} P_{\mathcal{T}}\right)=R\left(P_{\mathcal{S}} A^{*}\right)$ and $R\left(P_{\mathcal{T}} A P_{\mathcal{S}}\right)=R\left(P_{\mathcal{T}} A\right)$.

3. $\mathcal{H}=\mathcal{S}+\left(A^{*} \mathcal{T}\right)^{\perp}$ and $\mathcal{K}=\mathcal{T}+(A \mathcal{S})^{\perp}$.

Proof. $1 \Rightarrow 2$. Let $E, F$ be two bounded projections with $R(E)=\mathcal{S}$ and $R(F)=\mathcal{T}$ such that $A E=$ $F^{*} A$. To prove item 2 it suffices to prove that $R\left(P_{\mathcal{S}} A^{*}\right) \subseteq R\left(P_{\mathcal{S}} A^{*} P_{\mathcal{T}}\right)$ and $R\left(P_{\mathcal{T}} A\right) \subseteq R\left(P_{\mathcal{T}} A P_{\mathcal{S}}\right)$. Let us see the second inclusion: $P_{\mathcal{T}} A=P_{\mathcal{T}} A E+P_{\mathcal{T}} A(I-E)=P_{\mathcal{T}} A P_{\mathcal{S}} E+P_{\mathcal{T}}\left(I-F^{*}\right) A=P_{\mathcal{T}} A P_{\mathcal{S}} E$ because $P_{\mathcal{T}} F^{*}=P_{\mathcal{T}}$. Then $R\left(P_{\mathcal{T}} A\right) \subseteq R\left(P_{\mathcal{T}} A P_{\mathcal{S}}\right)$. Similarly, $R\left(P_{\mathcal{S}} A^{*}\right) \subseteq R\left(P_{\mathcal{S}} A^{*} P_{\mathcal{T}}\right)$.

$2 \Rightarrow 3$. Let $y=A x \in R(A)$. Then, $A x=P_{\mathcal{T}} A x+P_{\mathcal{T} \perp} A x=P_{\mathcal{T}} A s+P_{\mathcal{T} \perp} A x$, for some $s \in \mathcal{S}$ because $R\left(P_{\mathcal{T}} A P_{\mathcal{S}}\right)=R\left(P_{\mathcal{T}} A\right)$. Thus, $A x=A s-P_{\mathcal{T}^{\perp}} A s+P_{\mathcal{T}^{\perp}} A x \in A \mathcal{S}+\mathcal{T}^{\perp}$. Therefore, $R(A) \subseteq A \mathcal{S}+\mathcal{T}^{\perp}$. Then $\mathcal{H}=A^{-1}\left(A \mathcal{S}+\mathcal{T}^{\perp}\right)=\mathcal{S}+A^{-1}\left(\mathcal{T}^{\perp}\right)$. The proof of the second equality is similar.

$3 \Rightarrow 1$. If $\mathcal{H}=\mathcal{S}+\left(A^{*} \mathcal{T}\right)^{\perp}$ and $\mathcal{K}=\mathcal{T}+(A \mathcal{S})^{\perp}$ then there exist bounded projections $E$ and $F$ with $R(E)=\mathcal{S}, N(E) \subseteq\left(A^{*} \mathcal{T}\right)^{\perp}, R(F)=\mathcal{T}$ and $N(F) \subseteq(A \mathcal{S})^{\perp}$. We claim that $A E=F^{*} A$. In fact, $F^{*} A s=A s=A E s$ for all $s \in \mathcal{S}$ and $F^{*} A x=0=A E x$ for all $x \in N(E)$.

The following is a necessary condition for complementability.

Lemma 2.6. If $A$ is $(\mathcal{S}, \mathcal{T})$-complementable then $(A \mathcal{S})^{\perp} \cap \mathcal{T}=N\left(A^{*}\right) \cap \mathcal{T}$ and $\left(A^{*} \mathcal{T}\right)^{\perp} \cap \mathcal{S}=$ $N(A) \cap \mathcal{S}$.

Proof. Assume that $A$ is $(\mathcal{S}, \mathcal{T})$-complementable and let $E, F$ be projections onto $\mathcal{S}, \mathcal{T}$ respectively, such that $A E=F^{*} A$. Thus, if $x \in(A \mathcal{S})^{\perp} \cap \mathcal{T}$ then, using that $(A \mathcal{S})^{\perp}=\left(A^{*}\right)^{-1}\left(\mathcal{S}^{\perp}\right), A^{*} x \in \mathcal{S}^{\perp}$ and $x \in \mathcal{T}$, and so $\left\langle A^{*} x, y\right\rangle=\left\langle A^{*} F x, y\right\rangle=\left\langle E^{*} A^{*} x, y\right\rangle=\left\langle A^{*} x, E y\right\rangle=0$ for all $y \in \mathcal{H}$. Therefore, $A^{*} x=0$ and so $x \in N\left(A^{*}\right) \cap \mathcal{T}$. Since $(A \mathcal{S})^{\perp} \cap \mathcal{T} \supseteq N\left(A^{*}\right) \cap \mathcal{T}$ always holds, the equality is proved. Similarly, it can be proved that $\left(A^{*} \mathcal{T}\right)^{\perp} \cap \mathcal{S}=N(A) \cap \mathcal{S}$. 
Corollary 2.7. $A$ is $(\mathcal{S}, \mathcal{T})$-complementable if and only if $R(A)=A \mathcal{S} \dot{+} \mathcal{T}^{\perp} \cap R(A)$ and $R\left(A^{*}\right)=$ $A^{*} \mathcal{T}+\mathcal{S}^{\perp} \cap R\left(A^{*}\right)$.

Proof. For any $A \in L(\mathcal{H}, \mathcal{K})$, the equality $\left(A^{*} \mathcal{T}\right)^{\perp} \cap \mathcal{S}=N(A) \cap \mathcal{S}$ holds if and only if $A \mathcal{S} \cap \mathcal{T}^{\perp}=\{0\}$. In fact, suppose that $A \mathcal{S} \cap \mathcal{T}^{\perp}=\{0\}$ and let $x \in\left(A^{*} \mathcal{T}\right)^{\perp} \cap \mathcal{S}$ then $A x \in A \mathcal{S} \cap \mathcal{T}^{\perp}=\{0\}$. Then $x \in N(A)$. The converse is similar.

If $A$ is $(\mathcal{S}, \mathcal{T})$-complementable then, by Proposition $2.5, \mathcal{K}=\mathcal{T}+(A \mathcal{S})^{\perp}$ and, by Lemma 2.6, $(A \mathcal{S})^{\perp} \cap \mathcal{T}=N\left(A^{*}\right) \cap \mathcal{T}$. Thus, $R\left(A^{*}\right)=A^{*} \mathcal{T}+\mathcal{S}^{\perp} \cap R\left(A^{*}\right)$ and $A^{*} \mathcal{T} \cap \mathcal{S}^{\perp}=\{0\}$ by the previous comment. The proof of the second equality is similar.

For the converse, suppose that $R(A)=A \mathcal{S}+\mathcal{T}^{\perp} \cap R(A)$. Hence, $\mathcal{H}=A^{-1}\left(A \mathcal{S}+\mathcal{T}^{\perp} \cap R(A)\right)=$ $\mathcal{S}+N(A)+A^{-1}\left(\mathcal{T}^{\perp}\right)=\mathcal{S}+A^{-1}\left(\mathcal{T}^{\perp}\right)$. Analogously, from $R\left(A^{*}\right)=A^{*} \mathcal{T} \dot{+}\left(\mathcal{S}^{\perp} \cap R\left(A^{*}\right)\right)$, we get that $\mathcal{K}=\mathcal{T}+(A \mathcal{S})^{\perp}$, and the proof is complete.

By Proposition 2.5, if $A=I \in L(\mathcal{H})$ and $\mathcal{S}, \mathcal{T}$ are two closed subspaces of $\mathcal{H}$ then $A$ is $(\mathcal{S}, \mathcal{T})$-complementable if and only if $\mathcal{H}=\mathcal{S}+\mathcal{T}^{\perp}$. By [6, Theorem 1], $\mathcal{H}=\mathcal{S}+\mathcal{T}^{\perp}$ if and only if $\left\|P_{\mathcal{S}}-P_{\mathcal{T}}\right\|<1$. More generally, if $A$ has closed range or $A$ is invertible, the complementability can be characterized as follows:

Proposition 2.8. Given $A \in L(\mathcal{H}, \mathcal{K})$ with closed range, the following conditions are equivalent:

1. A is $(\mathcal{S}, \mathcal{T})$-complementable.

2. $\mathcal{H}=\mathcal{S}+\left(A^{*} \mathcal{T}\right)^{\perp}, A^{*}(\mathcal{T})$ is closed and $\left(A^{*} \mathcal{T}\right)^{\perp} \cap \mathcal{S}=N(A) \cap \mathcal{S}$.

3. $\mathcal{K}=\mathcal{T}+(A \mathcal{S})^{\perp}, A(\mathcal{S})$ is closed and $(A \mathcal{S})^{\perp} \cap \mathcal{T}=N\left(A^{*}\right) \cap \mathcal{T}$.

Proof. $1 \Leftrightarrow 2$. Assume that $A$ is $(\mathcal{S}, \mathcal{T})$-complementable. We only need to prove that $A^{*}(\mathcal{T})$ is closed. Now, by Corollary 2.7, $R\left(A^{*}\right)=A^{*}(\mathcal{T}) \dot{+} \mathcal{S}^{\perp} \cap R\left(A^{*}\right)$ and, since $R\left(A^{*}\right)$ is closed, we get that $A^{*}(\mathcal{T})$ is closed because of [32, Theorem 5.10].

Conversely, suppose that $\mathcal{H}=\mathcal{S}+\left(A^{*} \mathcal{T}\right)^{\perp}, A^{*}(\mathcal{T})$ is closed and $\left(A^{*} \mathcal{T}\right)^{\perp} \cap \mathcal{S}=N(A) \cap \mathcal{S}=: \mathcal{N}$. We shall prove that $\mathcal{K}=\mathcal{T}+(A \mathcal{S})^{\perp}$. Now, $\mathcal{H}=(\mathcal{S} \ominus \mathcal{N}) \dot{+}\left(A^{*} \mathcal{T}\right)^{\perp}$ or, equivalently, $\mathcal{H}=$ $(\mathcal{S} \ominus \mathcal{N})^{\perp} \dot{+} A^{*} \mathcal{T}$. Therefore, if $y \in R\left(A^{*}\right)$, then $y=s+A^{*} t$ for some $s \in(\mathcal{S} \ominus \mathcal{N})^{\perp}$ and $t \in \mathcal{T}$; and so $s \in(\mathcal{S} \ominus \mathcal{N})^{\perp} \cap R\left(A^{*}\right)$. Hence, $R\left(A^{*}\right)=(\mathcal{S} \ominus \mathcal{N})^{\perp} \cap R\left(A^{*}\right) \dot{+} A^{*} \mathcal{T}$. From this, $\mathcal{K}=$ $\left(A^{*}\right)^{-1}\left((\mathcal{S} \ominus \mathcal{N})^{\perp}\right)+\mathcal{T}=(A(\mathcal{S} \ominus \mathcal{N}))^{\perp}+\mathcal{T}=(A \mathcal{S})^{\perp}+\mathcal{T}$. Therefore, $A$ is $(\mathcal{S}, \mathcal{T})$-complementable

The equivalence $1 \Leftrightarrow 3$ can be proved similarly.

Corollary 2.9. Let $A \in L(\mathcal{H}, \mathcal{K})$ be an invertible operator. The next conditions are equivalent:

1. $A$ is $(\mathcal{S}, \mathcal{T})$-complementable

2. $\mathcal{H}=\mathcal{S} \dot{+}\left(A^{*} \mathcal{T}\right)^{\perp}$

3. $\mathcal{K}=\mathcal{T} \dot{+}(A \mathcal{S})^{\perp}$.

4. $\left\|P_{\mathcal{S}}-P_{\left(A^{*} \mathcal{T}\right)^{\perp}}\right\|<1$.

5. $\left\|P_{\mathcal{T}}-P_{(A \mathcal{S}) \perp}\right\|<1$.

The next result shows some equivalent conditions for complementability when $A=P_{\mathcal{M}}$, where $\mathcal{M}$ is a a closed subspace of $\mathcal{H}$.

Proposition 2.10. Let $\mathcal{M} \subseteq \mathcal{H}$ be a closed subspace. The next conditions are equivalent:

1. $P_{\mathcal{M}}$ is $(\mathcal{S}, \mathcal{T})$-complementable 
2. $\mathcal{M}=\left(\mathcal{S}+\mathcal{M}^{\perp}\right) \cap \mathcal{M}+\mathcal{T}^{\perp} \cap \mathcal{M}$, and $\mathcal{T}+\mathcal{M}^{\perp}$ is closed.

3. $\mathcal{H}=\mathcal{S}+\mathcal{M}^{\perp}+\mathcal{T}^{\perp} \cap \mathcal{M}=\mathcal{T}+\mathcal{M}^{\perp}+\mathcal{S}^{\perp} \cap \mathcal{M}$.

4. The subspaces $P_{\mathcal{M}} \mathcal{S}$ and $P_{\mathcal{M}} \mathcal{T}$ are closed and the Grammian operator $G: P_{\mathcal{M}} \mathcal{S} \rightarrow P_{\mathcal{M}} \mathcal{T}$, $G:=\left.P_{P_{\mathcal{M}} \mathcal{T}}\right|_{P_{\mathcal{M}} \mathcal{S}}$ is invertible.

Proof. It always holds that $P_{\mathcal{M}} \mathcal{S}=\left(\mathcal{S}+\mathcal{M}^{\perp}\right) \cap \mathcal{M}$ : if $x \in P_{\mathcal{M}} \mathcal{S}$, there exists $s \in \mathcal{S}$ such that $x=P_{\mathcal{M}} s$. Then $s=x+P_{\mathcal{M}^{\perp}} s$, or $x=s-P_{\mathcal{M}^{\perp}} s \in \mathcal{S}+\mathcal{M}^{\perp}$. Then $x \in\left(\mathcal{S}+\mathcal{M}^{\perp}\right) \cap \mathcal{M}$. Conversely, if $x \in\left(\mathcal{S}+\mathcal{M}^{\perp}\right) \cap \mathcal{M}$ then $x=s+m^{\perp}$, for some $s \in \mathcal{S}, m^{\perp} \in \mathcal{M}^{\perp}$. Then $x=P_{\mathcal{M}} x=P_{\mathcal{M}} s \in P_{\mathcal{M}} \mathcal{S}$.

$1 \Rightarrow 2$. If $P_{\mathcal{M}}$ is $(\mathcal{S}, \mathcal{T})$-complementable then, by Corollary 2.7 and [32, Theorem 5.10], $P_{\mathcal{M}} \mathcal{S}$ and $P_{\mathcal{M}} \mathcal{T}$ are closed and $\mathcal{M}=P_{\mathcal{M}} \mathcal{S}+\mathcal{T}^{\perp} \cap \mathcal{M}$. Then, $\mathcal{M}=\left(\mathcal{S}+\mathcal{M}^{\perp}\right) \cap \mathcal{M} \dot{+} \mathcal{T}^{\perp} \cap \mathcal{M}$ and $\mathcal{T}+\mathcal{M}^{\perp}$ is closed because of Theorem 2.1.

$2 \Rightarrow 3$. If $\mathcal{M}=\left(\mathcal{S}+\mathcal{M}^{\perp}\right) \cap \mathcal{M} \dot{+} \mathcal{T}^{\perp} \cap \mathcal{M}$ then $\mathcal{H}=\left(\mathcal{S}+\mathcal{M}^{\perp}\right) \cap \mathcal{M} \dot{+} \mathcal{T}^{\perp} \cap \mathcal{M}+\mathcal{M}^{\perp} \subseteq$ $\mathcal{S}+\mathcal{M}^{\perp}+\mathcal{T}^{\perp} \cap \mathcal{M}$, as desired. To prove the second equality it suffices to check that by taking orthogonal complement in the Hilbert space $\mathcal{M}$ in the equality $\mathcal{M}=P_{\mathcal{M}} \mathcal{S}+\mathcal{T}^{\perp} \cap \mathcal{M}$, and using that $\mathcal{T}+\mathcal{M}^{\perp}$ is closed, we get that $\mathcal{M}=\left(P_{\mathcal{M}} \mathcal{S}\right)^{\perp} \cap \mathcal{M} \dot{+}\left(\mathcal{T}+\mathcal{M}^{\perp}\right) \cap \mathcal{M}$. But $\left(P_{\mathcal{M}} \mathcal{S}\right)^{\perp}=N\left(P_{\mathcal{S}} P_{\mathcal{M}}\right)=$ $\left(\mathcal{M} \cap \mathcal{S}^{\perp}\right) \oplus \mathcal{M}^{\perp}$. Therefore, $\mathcal{M}=\mathcal{S}^{\perp} \cap \mathcal{M} \dot{+}\left(\mathcal{T}+\mathcal{M}^{\perp}\right) \cap \mathcal{M}$. Proceeding as before, we get that $\mathcal{H}=\mathcal{T}+\mathcal{M}^{\perp}+\mathcal{S}^{\perp} \cap \mathcal{M}$.

$3 \Rightarrow 1$. Assume that $\mathcal{H}=\mathcal{S}+\mathcal{M}^{\perp}+\mathcal{T}^{\perp} \cap \mathcal{M}$. Now, $\mathcal{M}^{\perp}+\mathcal{T}^{\perp} \cap \mathcal{M}=N\left(P_{\mathcal{T}} P_{\mathcal{M}}\right)=R\left(P_{\mathcal{M}} P_{\mathcal{T}}\right)^{\perp}=$ $\left(P_{\mathcal{M}} \mathcal{T}\right)^{\perp}$. Thus, $\mathcal{H}=\mathcal{S}+\left(P_{\mathcal{M}} \mathcal{T}\right)^{\perp}$. The equality $\mathcal{H}=\mathcal{T}+\left(P_{\mathcal{M}} \mathcal{S}\right)^{\perp}$ follows in the same way from $\mathcal{H}=\mathcal{T}+\mathcal{M}^{\perp}+\mathcal{S}^{\perp} \cap \mathcal{M}$.

$2 \Leftrightarrow$ 4. Suppose that $\mathcal{M}=\left(\mathcal{S}+\mathcal{M}^{\perp}\right) \cap \mathcal{M} \dot{+} \mathcal{T}^{\perp} \cap \mathcal{M}$, and $\mathcal{T}+\mathcal{M}^{\perp}$ is closed. From this, $P_{\mathcal{M}} \mathcal{S}$ and $P_{\mathcal{M}} \mathcal{T}$ are closed subspaces. Let us prove that $G$ is invertible. Consider $y \in P_{\mathcal{M}} \mathcal{T}$. Then, $y \in \mathcal{M}$ and so $y=x_{1}+x_{2}$ for some $x_{1} \in\left(\mathcal{S}+\mathcal{M}^{\perp}\right) \cap \mathcal{M}=P_{\mathcal{M}} \mathcal{S}$ and $x_{2} \in \mathcal{T}^{\perp} \cap \mathcal{M}$. Hence, since $\mathcal{T}^{\perp} \cap \mathcal{M} \subseteq\left(P_{\mathcal{M}} \mathcal{T}\right)^{\perp}$ we get that $G x_{1}=P_{P_{\mathcal{M}} \mathcal{T} x_{1}}=P_{P_{\mathcal{M}} \mathcal{T}} y=y$. Thus, $G$ is surjective. On the other side, let $x \in P_{\mathcal{M}} \mathcal{S}$ such that $G x=0$. Then, $x \in\left(P_{\mathcal{M}} \mathcal{T}\right)^{\perp} \cap P_{\mathcal{M}} \mathcal{S}=\left(\mathcal{T}^{\perp} \cap \mathcal{M}\right) \cap P_{\mathcal{M}} \mathcal{S}=\{0\}$. Therefore $G$ is injective, so that $G$ is invertible.

Conversely, assume that $P_{\mathcal{M}} \mathcal{S}$ and $P_{\mathcal{M}} \mathcal{T}$ are closed subspaces and $G:=\left.P_{P_{\mathcal{M}} \mathcal{T}}\right|_{P_{\mathcal{M}} \mathcal{S}}$ is invertible. Then, by [24, Proposition 2.1], $\left(P_{\mathcal{M}} \mathcal{T}\right)^{\perp}+P_{\mathcal{M}} \mathcal{S}=\mathcal{H}$. Hence, $\mathcal{M}=P_{\mathcal{M}}\left(\left(P_{\mathcal{M}} \mathcal{T}\right)^{\perp}\right)+P_{\mathcal{M}} \mathcal{S}$. Now, as $P_{\mathcal{M}} \mathcal{T}=\left(\mathcal{T}+\mathcal{M}^{\perp}\right) \cap \mathcal{M}$ a simple computation shows that $P_{\mathcal{M}}\left(\left(P_{\mathcal{M}} \mathcal{T}\right)^{\perp}\right)=\mathcal{T}^{\perp} \cap \mathcal{M}$. The proof is complete.

By the proof of Proposition 2.5, the set $\mathcal{P}(A, \mathcal{S}, \mathcal{T})$ can be characterized in the following way:

Proposition 2.11. Let $A$ be $(\mathcal{S}, \mathcal{T})$-complementable. Then

$$
\mathcal{P}(A, \mathcal{S}, \mathcal{T})=\left\{(E, F) \in \mathcal{Q} \times \mathcal{Q}: R(E)=\mathcal{S}, N(E) \subseteq\left(A^{*} \mathcal{T}\right)^{\perp}, R(F)=\mathcal{T} \text { and } N(F) \subseteq(A \mathcal{S})^{\perp}\right\}
$$

If $A$ is $(\mathcal{S}, \mathcal{T})$-complementable, consider $\mathcal{N}=\mathcal{S} \cap N(A)$ and $\mathcal{N}^{*}=\mathcal{T} \cap N\left(A^{*}\right)$. By Lemma 2.6, $\mathcal{H}=\mathcal{S} \dot{+}\left(A^{*} \mathcal{T}\right)^{\perp} \ominus \mathcal{N}$ and $\mathcal{K}=\mathcal{T} \dot{+}(A \mathcal{S})^{\perp} \ominus \mathcal{N}^{*}$ and the corresponding projections

$$
\tilde{E}=Q_{\mathcal{S} / /\left(A^{*} \mathcal{T}\right)^{\perp} \ominus \mathcal{N}} \text { and } \tilde{F}=Q_{\mathcal{T} / /(A \mathcal{S})^{\perp} \ominus \mathcal{N}^{*}}
$$

are such that $(\tilde{E}, \tilde{F}) \in \mathcal{P}(A, \mathcal{S}, \mathcal{T})$. Moreover, the set $\mathcal{P}(A, \mathcal{S}, \mathcal{T})$ is an affine manifold as the next corollary shows:

Corollary 2.12. Let $A$ be $(\mathcal{S}, \mathcal{T})$-complementable. Then

$$
\mathcal{P}(A, \mathcal{S}, \mathcal{T})=(\tilde{E}, \tilde{F})+L\left(S^{\perp}, \mathcal{N}\right) \times L\left(\mathcal{T}^{\perp}, \mathcal{N}^{*}\right),
$$


where $\tilde{E}$ and $\tilde{F}$ are the projections given by (1) and $L\left(S^{\perp}, \mathcal{N}\right), L\left(\mathcal{T}^{\perp}, \mathcal{N}^{*}\right)$ are viewed as subspaces of $L(\mathcal{H}), L(\mathcal{K})$, respectively. In particular, $\mathcal{P}(A, \mathcal{S}, \mathcal{T})=\{(\tilde{E}, \tilde{F})\}$ if and only if $\mathcal{S} \cap N(A)=\{0\}$ and $\mathcal{T} \cap N\left(A^{*}\right)=\{0\}$.

Given two closed subspaces $\mathcal{S}, \mathcal{T}$ of $\mathcal{H}, \mathcal{K}$, respectively, we identify each $A \in L(\mathcal{H}, \mathcal{K})$ with the matrix

$$
A=\underset{\mathcal{T}^{\perp}}{\mathcal{T}}\left(\begin{array}{ll}
A_{11} & A_{12} \\
A_{21} & A_{22}
\end{array}\right) \stackrel{\mathcal{S}}{\mathcal{S}}
$$

where $A_{11}=\left.P_{\mathcal{T}} A\right|_{\mathcal{S}}, A_{12}=\left.P_{\mathcal{T}} A\right|_{\mathcal{S}^{\perp}}, A_{21}=\left.P_{\mathcal{T}^{\perp}} A\right|_{\mathcal{S}}, A_{22}=\left.P_{\mathcal{T} \perp} A\right|_{\mathcal{S}^{\perp}}$. The next result provides a characterization of the $(\mathcal{S}, \mathcal{T})$-complementability in terms of the matrix representation of $A$ and $A^{*}$. The first part of the following result can be also found in [4, Proposition 3.2]

Corollary 2.13. Consider the matrix representation (2). Then, $A$ is $(\mathcal{S}, \mathcal{T})$-complementable if and only if $R\left(A_{12}\right) \subseteq R\left(A_{11}\right)$ and $R\left(A_{21}^{*}\right) \subseteq R\left(A_{11}^{*}\right)$. In this case,

$$
\mathcal{P}(A, \mathcal{S}, \mathcal{T})=\left\{(E, F): E=\left(\begin{array}{cc}
1 & X \\
0 & 0
\end{array}\right) \underset{\mathcal{S}^{\perp}}{\mathcal{S}} \text { and } F=\left(\begin{array}{cc}
1 & Y \\
0 & 0
\end{array}\right) \underset{\mathcal{T}^{\perp}}{\mathcal{T}} \text { with } A_{11} X=A_{12}, A_{11}^{*} Y=A_{21}^{*}\right\}
$$

Proof. From Proposition 2.5, $A$ is $(\mathcal{S}, \mathcal{T})$-complementable if and only if $R\left(P_{\mathcal{S}} A^{*} P_{\mathcal{T}}\right)=R\left(P_{\mathcal{S}} A^{*}\right)$ and $R\left(P_{\mathcal{T}} A P_{\mathcal{S}}\right)=R\left(P_{\mathcal{T}} A\right)$. But these last equalities hold if and only if $R\left(P_{\mathcal{S}} A^{*} P_{\mathcal{T} \perp}\right) \subseteq R\left(P_{\mathcal{S}} A^{*} P_{\mathcal{T}}\right)$ and $R\left(P_{\mathcal{T}} A P_{\mathcal{S}^{\perp}}\right) \subseteq R\left(P_{\mathcal{T}} A P_{\mathcal{S}}\right)$, which proves the assertion. In this case, by Douglas' inclusion operator range theorem [17, Theorem 1], there exist $X \in L\left(\mathcal{S}^{\perp}, \mathcal{S}\right)$ and $Y \in L\left(\mathcal{T}^{\perp}, \mathcal{T}\right)$ such that $A_{11} X=A_{12}$ and $A_{11}^{*} Y=A_{21}^{*}$. Define $E=\left(\begin{array}{cc}1 & X \\ 0 & 0\end{array}\right)$ and $F=\left(\begin{array}{cc}1 & Y \\ 0 & 0\end{array}\right)$; clearly, $E, F$ are projections onto $\mathcal{S}, \mathcal{T}$, respectively and it is straightforward that $A E=F^{*} A$. Therefore, $(E, F) \in \mathcal{P}(A, \mathcal{S}, \mathcal{T})$. The other inclusion follows in a similar way.

\subsection{Weak complementability}

By considering the adjoints of $E$ and $F$ in Definition 2.2, the definition of complementability can be rewritten in the following way: $A \in L(\mathcal{H}, \mathcal{K})$ is $(\mathcal{S}, \mathcal{T})$-complementable if there exist two bounded projections $E, F$ with $N(E)=\mathcal{S}^{\perp}$ and $N(F)=\mathcal{T}^{\perp}$ such that $E A^{*}=(F A)^{*}$. Motivated by this fact, we introduce now a weaker notion:

Definition 2.14. An operator $A \in L(\mathcal{H}, \mathcal{K})$ is called $(\mathcal{S}, \mathcal{T})$-weakly complementable if there exist two densely defined projections $E, F$ with $N(E)=\mathcal{S}^{\perp}$ and $N(F)=\mathcal{T}^{\perp}$ such that:

a) $E\left|P_{\mathcal{T}} A P_{\mathcal{S}}\right|^{1 / 2}$ and $F\left|P_{\mathcal{S}} A^{*} P_{\mathcal{T}}\right|^{1 / 2}$ are bounded operators;

b) $F A \in L(\mathcal{H}, \mathcal{K}), E A^{*} \in L(\mathcal{K}, \mathcal{H})$ and $E A^{*}=(F A)^{*}$.

The concept of weakly complementability was first defined by Antezana et al. in [4] with the purpose of extending the notion of shorted operator to bounded linear operators between two different Hilbert spaces. Their definition relies on the matricial form of $A$ in terms of $\mathcal{S}$ and $\mathcal{T}$ as in (2). Next, we prove that our definition coincides with that of [4].

Proposition 2.15. If $A$ has the matrix representation given by (2), then the following conditions are equivalent:

1. A is $(\mathcal{S}, \mathcal{T})$-weakly complementable. 
2. $R\left(A_{12}\right) \subseteq R\left(\left|A_{11}^{*}\right|^{1 / 2}\right)$ and $R\left(A_{21}^{*}\right) \subseteq R\left(\left|A_{11}\right|^{1 / 2}\right)$.

Proof. $1 \Rightarrow 2$. Suppose that $A$ is $(\mathcal{S}, \mathcal{T})$-weakly complementable and let $E, F$ two densely defined projections with $N(E)=\mathcal{S}^{\perp}$ and $N(F)=\mathcal{T}^{\perp}$ such that conditions $a$ ) and $b$ ) of Definition 2.14 hold. Hence, if $E=\left(\begin{array}{cc}I & 0 \\ X & 0\end{array}\right) \underset{\mathcal{S}^{\perp}}{\mathcal{S}}$ and $F=\left(\begin{array}{cc}I & 0 \\ Y & 0\end{array}\right) \mathcal{T}^{\perp}$, then from condition a) the operators $X\left|A_{11}\right|^{1 / 2}$ and $Y\left|A_{11}^{*}\right|^{1 / 2}$ are bounded. On the other hand, by condition $b$ ), we obtain that $X A_{11}^{*}=A_{12}^{*}$ and $\left(Y A_{11}\right)^{*}=A_{21}^{*}$. Let $A_{11}^{*}=U^{*}\left|A_{11}^{*}\right|$ be the polar decomposition of $A_{11}^{*}$ where $U$ is the partial isometry with $N(U)=N\left(A_{11}\right)$. Then, $A_{12}^{*}=X A_{11}^{*}=X\left|A_{11}\right|^{1 / 2} U^{*}\left|A_{11}^{*}\right|^{1 / 2} \in L\left(\mathcal{T}, \mathcal{S}^{\perp}\right)$ and since $Z:=X\left|A_{11}\right|^{1 / 2} U^{*} \in L\left(\mathcal{S}, \mathcal{T}^{\perp}\right)$, we have that $A_{12}=\left|A_{11}^{*}\right|^{1 / 2} Z^{*}$ and so $R\left(A_{12}\right) \subseteq R\left(\left|A_{11}^{*}\right|^{1 / 2}\right)$. In a similar way, from $A_{21}=Y A_{11}=Y\left|A_{11}^{*}\right|^{1 / 2} U\left|A_{11}\right|^{1 / 2} \in L\left(\mathcal{S}, \mathcal{T}^{\perp}\right)$ we get that $R\left(A_{21}^{*}\right) \subseteq R\left(\left|A_{11}\right|^{1 / 2}\right)$ as desired.

$2 \Rightarrow 1$. Assume that $R\left(A_{12}\right) \subseteq R\left(\left|A_{11}^{*}\right|^{1 / 2}\right)$ and $R\left(A_{21}^{*}\right) \subseteq R\left(\left|A_{11}\right|^{1 / 2}\right)$. Then, by Douglas' theorem [17, Theorem 1], there exist operators $B, C$ such that $A_{12}=\left|A_{11}^{*}\right|^{1 / 2} B, R(B) \subseteq \overline{R\left(A_{11}\right)}$ and $A_{21}^{*}=\left|A_{11}\right|^{1 / 2} C$ and $R(C) \subseteq \overline{R\left(A_{11}^{*}\right)}$. Therefore,

$$
A=\underset{\mathcal{T}^{\perp}}{\mathcal{T}}\left(\begin{array}{cc}
\left|A_{11}^{*}\right| U & \left|A_{11}^{*}\right|^{1 / 2} B \\
C^{*}\left|A_{11}\right|^{1 / 2} & A_{22}
\end{array}\right) \underset{\mathcal{S}^{\perp}}{\mathcal{S}}
$$

where $A_{11}^{*}=U^{*}\left|A_{11}^{*}\right|=\left|A_{11}\right| U^{*}$ is the polar decomposition of $A_{11}^{*}$.

Define: $\quad E_{0}=\stackrel{\mathcal{S}}{\mathcal{S}} \mathcal{S}^{\perp}\left(\begin{array}{cc}I & 0 \\ B^{*} U\left(\left|A_{11}\right|^{1 / 2}\right)^{\dagger} & 0\end{array}\right) \stackrel{\mathcal{S}}{\mathcal{S}}$ and $F_{0}={ }_{\mathcal{T}^{\perp}}^{\mathcal{T}}\left(\begin{array}{cc}I & 0 \\ C^{*} U^{*}\left(\left|A_{11}^{*}\right|^{1 / 2}\right)^{\dagger} & 0\end{array}\right) \underset{\mathcal{T}^{\perp}}{\mathcal{T}}$. Then, $E_{0}, F_{0}$ are two densely defined projections, $\mathcal{D}\left(E_{0}\right)=\mathcal{D}\left(\left(\left|A_{11}\right|^{1 / 2}\right)^{\dagger}\right)+\mathcal{S}^{\perp}$ and $\mathcal{D}\left(F_{0}\right)=\mathcal{D}\left(\left(\left|A_{11}^{*}\right|^{1 / 2}\right)^{\dagger}+\right.$ $\mathcal{T}^{\perp}$, with $N\left(E_{0}\right)=\mathcal{S}^{\perp}$ and $N\left(F_{0}\right)=\mathcal{T}^{\perp}$. Thus, since $\left|P_{\mathcal{T}} A P_{\mathcal{S}}\right|=\left|A_{11}\right|$ and $\left|P_{\mathcal{S}} A^{*} P_{\mathcal{T}}\right|=\left|A_{11}^{*}\right|$ then $E_{0}\left|P_{\mathcal{T}} A P_{\mathcal{S}}\right|^{1 / 2}=\left(\begin{array}{cc}\left|A_{11}\right|^{1 / 2} & 0 \\ B^{*} U P_{\overline{R\left(\left|A_{11}\right|^{1 / 2}\right)}} & 0\end{array}\right) \in L(\mathcal{H}), F_{0}\left|P_{\mathcal{S}} A^{*} P_{\mathcal{T}}\right|^{1 / 2}=\left(\begin{array}{cc}\left|A_{11}^{*}\right|^{1 / 2} & 0 \\ C^{*} U^{*} P_{\overline{R\left(\left|A_{11}^{*}\right|^{1 / 2}\right)}} & 0\end{array}\right) \in L(\mathcal{K})$ and $E_{0} A^{*}=\left(\begin{array}{cc}A_{11}^{*} & A_{21}^{*} \\ A_{12}^{*} & B^{*} U C\end{array}\right)=\left(F_{0} A\right)^{*} \in L(\mathcal{H}, \mathcal{K})$, where we used that $U^{*}\left|A_{11}^{*}\right|^{1 / 2}=\left|A_{11}\right|^{1 / 2} U^{*}$. Therefore, $A$ is $(\mathcal{S}, \mathcal{T})$-weakly complementable.

Notice that every positive operator in $L(\mathcal{H})$ is $\mathcal{S}$-weakly complementable for every closed subspace $\mathcal{S}$ of $\mathcal{H}$ : in fact, if $A=\left(\begin{array}{ll}A_{11} & A_{12} \\ A_{12}^{*} & A_{22}\end{array}\right) \stackrel{\mathcal{S}}{\mathcal{S}}$ 鿆 the matrix representation of $A$ then $A_{11} \geq 0$ and $R\left(A_{12}\right)$ is always contained in $R\left(A_{11}^{1 / 2}\right)$.

Remark 2.16. Taking into account the proof of the above result we observe that if $A=\left(\begin{array}{ll}A_{11} & A_{12} \\ A_{21} & A_{22}\end{array}\right)$ is $(\mathcal{S}, \mathcal{T})$-weakly complementable then

$$
A=\left(\begin{array}{cc}
\left|A_{11}^{*}\right| U & \left|A_{11}^{*}\right|^{1 / 2} B \\
C^{*}\left|A_{11}\right|^{1 / 2} & A_{22}
\end{array}\right)
$$

where $A_{11}^{*}=U^{*}\left|A_{11}^{*}\right|$ is the polar decomposition of $A_{11}^{*}, B=\left(\left|A_{11}^{*}\right|^{1 / 2}\right)^{\dagger} A_{12}$ and $C=\left(\left|A_{11}\right|^{1 / 2}\right)^{\dagger} A_{21}^{*}$. Moreover,

$$
E_{0}=\left(\begin{array}{cc}
I & 0 \\
B^{*} U\left(\left|A_{11}\right|^{1 / 2}\right)^{\dagger} & 0
\end{array}\right) \stackrel{\mathcal{S}}{\mathcal{S}^{\perp}}
$$

and

$$
F_{0}=\left(\begin{array}{cc}
I & 0 \\
C^{*} U^{*}\left(\left|A_{11}^{*}\right|^{1 / 2}\right)^{\dagger} & 0
\end{array}\right) \stackrel{\mathcal{T}}{\mathcal{T}^{\perp}}
$$


are two densely defined projections with $\mathcal{D}\left(E_{0}\right)=\mathcal{D}\left(\left(\left|A_{11}\right|^{1 / 2}\right)^{\dagger}\right)+\mathcal{S}^{\perp}$ and $\mathcal{D}\left(F_{0}\right)=\mathcal{D}\left(\left(\left|A_{11}^{*}\right|^{1 / 2}\right)^{\dagger}+\right.$ $\mathcal{T}^{\perp}$, that verify the conditions of Definition 2.14. We remark that these projections are not closed, in general, but they are semiclosed operators in the sense given by Kaufman in [22]. He defined that an operator $C$ is semiclosed if there exists a bounded operator $B$ on $\mathcal{H}$, with range the domain of $C$, such that $C B$ is bounded. In our case, if we consider $\Gamma:=\left|A_{11}\right|^{1 / 2} P_{\mathcal{S}}+P_{N\left(\left|A_{11}\right|\right)}+P_{\mathcal{S}^{\perp}}$ then $\Gamma \in L(\mathcal{H}), R(\Gamma)=\mathcal{D}\left(E_{0}\right)$ and it is easy to check, using the formula for $E_{0}$, that $E_{0} \Gamma \in L(\mathcal{H})$, i.e., $E_{0}$ is semiclosed. Similarly, it can be proved that $F_{0}$ is semiclosed.

Finally, we highlight that if $A$ is $(\mathcal{S}, \mathcal{T})$-complementable then $E_{0}^{*}$ and $F_{0}^{*}$, are the projections defined by (1).

\section{Complementability and general Wiener-Hopf operators}

If $A \in L(\mathcal{H})$ and $\mathcal{S}$ is closed subspace of $\mathcal{H}$ then the operator $\left.P_{\mathcal{S}} A\right|_{\mathcal{S}}$, has been called by Devinatz and Shinbrot [15] a general Wiener-Hopf operator. It must be pointed out that this operator emerges as a generalization of the classical integral operator $g(x)=\int_{0}^{\infty} K(x-y) f(y) d y$ studied by Wiener, Hopf and others. Here, we shall consider the following more general definition:

Definition 3.1. Given two closed subspaces $\mathcal{S}, \mathcal{T}$ of $\mathcal{H}, \mathcal{K}$ respectively, the general Wiener-Hopf operator of $A \in L(\mathcal{H}, \mathcal{K}), T_{\mathcal{T}, \mathcal{S}}(A): \mathcal{S} \rightarrow \mathcal{T}$ is defined as

$$
T_{\mathcal{T}, \mathcal{S}}(A)=\left.P_{\mathcal{T}} A\right|_{\mathcal{S}}
$$

Using the matrix representation (2), it is clear that $T_{\mathcal{T}, \mathcal{S}}(A)=A_{11}$. There is an extensive literature concerning to the invertibility of the general Wiener-Hopf operator $\left.P_{\mathcal{S}} A\right|_{\mathcal{S}}$ see [15], [21],[29], [31], among others. Our aim in this section is to apply the previous results about complementability and weakly complementability to the study of the generalized invertibility (or, invertibility) of $T_{\mathcal{T}, \mathcal{S}}(A)$ and to obtain a formula for $T_{\mathcal{T}, \mathcal{S}}(A)^{\dagger}$ (or, $T_{\mathcal{T}, \mathcal{S}}(A)^{-1}$ ) in terms of the projections associated to these notions. Recall that for $A \in L(\mathcal{H}, \mathcal{K})$, the Moore-Penrose inverse of $A$ is the (non necessarily bounded) operator $A^{\dagger}: R(A) \oplus R(A)^{\perp} \rightarrow \mathcal{H}$, defined as $A^{\dagger}(A x+y)=x$ where $y \in R(A)^{\perp}$ and $x \in N(A)^{\perp}$.

Let us begin with the following result:

Theorem 3.2. If $A$ is $(\mathcal{S}, \mathcal{T})$-weakly complementable, then

$$
T_{\mathcal{T}, \mathcal{S}}(A)^{\dagger}=\left.\left(A P_{\mathcal{S}}\right)^{\dagger} F_{0} P_{\overline{R\left(T_{\mathcal{T}, \mathcal{S}}(A)\right)}}\right|_{\mathcal{D}\left(T_{\mathcal{T}, \mathcal{S}}(A)^{\dagger}\right)},
$$

where $F_{0}$ is the densely defined projection given by (5).

Proof. Assume that $A$ is $(\mathcal{S}, \mathcal{T})$-weakly complementable and let $F_{0}$ be the densely defined projection given by (5). Observe that, in this case, $T_{\mathcal{T}, \mathcal{S}}(A)^{\dagger}$ is not necessarily a bounded operator. Let us prove formula (6). For this, consider the matrix representations of $A$ and $F_{0}$ given in Remark 2.16 and $T:=P_{\mathcal{T}} A P_{\mathcal{S}}=\left(\begin{array}{cc}A_{11} & 0 \\ 0 & 0\end{array}\right)$. Therefore, a simple matrix computation shows that $A P_{\mathcal{S}}=$ $F_{0} A P_{\mathcal{S}}=F_{0} P_{T} A P_{\mathcal{S}}=F_{0} T$, where $F_{0} T$ is well defined since $R\left(A_{11}\right) \subseteq \mathcal{D}\left(\left(\left|A_{11}^{*}\right|^{1 / 2}\right)^{\dagger}\right)$. From

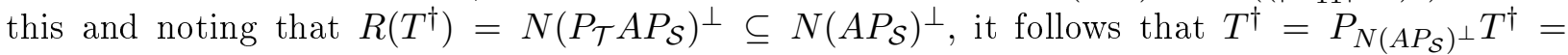
$\left(A P_{\mathcal{S}}\right)^{\dagger} A P_{\mathcal{S}} T^{\dagger}=\left(A P_{\mathcal{S}}\right)^{\dagger} F_{0} T T^{\dagger}=\left.\left(A P_{\mathcal{S}}\right)^{\dagger} F_{0} P_{\overline{R(T)}}\right|_{\mathcal{D}\left(T^{\dagger}\right)}$. Finally, notice that $\left.T^{\dagger}\right|_{\mathcal{T}}=T_{\mathcal{T}, \mathcal{S}}(A)^{\dagger}$, that $\overline{R(T)}=\overline{R\left(T_{\mathcal{T}, \mathcal{S}}(A)\right)}$ and $\mathcal{D}\left(T^{\dagger}\right) \cap \mathcal{T}=\mathcal{D}\left(T_{\mathcal{T}, \mathcal{S}}(A)^{\dagger}\right)$. The result is proved.

Given $T \in L(\mathcal{H}, \mathcal{K}), T^{\dagger} \in L(\mathcal{K}, \mathcal{H})$ if and only if $R(T)$ is closed. Hence, in what follows we provide conditions in terms of complementability that guarantee that $R\left(T_{\mathcal{T}, \mathcal{S}}(A)\right)$ is closed. For this purpose, we start with the next result. 
Lemma 3.3. $N\left(T_{\mathcal{T}, \mathcal{S}}(A)\right)=\mathcal{S} \cap N(A)$ if and only if $A(\mathcal{S}) \cap \mathcal{T}^{\perp}=\{0\}$.

Proof. The result follows noticing that $N\left(T_{\mathcal{T}, \mathcal{S}}(A)\right)=\mathcal{S} \cap A^{-1}\left(\mathcal{T}^{\perp}\right)$ and applying the remark at the beginning of the proof of Corollary 2.7 .

The following result follows by Theorem 2.1:

Proposition 3.4. If $T_{\mathcal{T}, \mathcal{S}}(A)$ has closed range then $A \mathcal{S}+\mathcal{T}^{\perp}$ is closed. If $A \mathcal{S}$ is closed, the converse holds.

Corollary 3.5. $T_{\mathcal{T}, \mathcal{S}}(A)$ has closed range and is injective if and only if $A(\mathcal{S})+\mathcal{T}^{\perp}$ is closed and $\mathcal{S} \cap N(A)=\{0\}$.

Proof. The nullspace of $T_{\mathcal{T}, \mathcal{S}}(A)$ is $N\left(T_{\mathcal{T}, \mathcal{S}}(A)\right)=\mathcal{S} \cap A^{-1}\left(\mathcal{T}^{\perp}\right)$.

Suppose that $T_{\mathcal{T}, \mathcal{S}}(A)$ has closed range and is injective. Then, by Proposition 3.4, $A \mathcal{S}+\mathcal{T}^{\perp}$ is closed. Since $\mathcal{S} \cap N(A) \subseteq \mathcal{S} \cap A^{-1}\left(\mathcal{T}^{\perp}\right)=\{0\}$, then $\mathcal{S} \cap N(A)=\mathcal{S} \cap A^{-1}\left(\mathcal{T}^{\perp}\right)=\{0\}$. Also, by the proof of Corollary 2.7, this implies that $A \mathcal{S} \cap \mathcal{T}^{\perp}=\{0\}$ so the sum is direct.

Conversely, suppose that $A(\mathcal{S}) \dot{+} \mathcal{T}^{\perp}$ is closed and $\mathcal{S} \cap N(A)=\{0\}$. Then, by [32, Theorem 5.10], $A(\mathcal{S})$ is closed. Applying Proposition 3.4, the range of $T_{\mathcal{T}, \mathcal{S}}(A)$ is closed. Since $A(\mathcal{S}) \cap \mathcal{T}^{\perp}=\{0\}$ then, by Lemma $3.3, T_{\mathcal{T}, \mathcal{S}}(A)$ is injective.

Theorem 3.6. The following conditions are equivalent:

1. A is $(\mathcal{S}, \mathcal{T})$-complementable and $A(\mathcal{S}), A^{*}(\mathcal{T})$ are closed subspaces.

2. $T_{\mathcal{T}, \mathcal{S}}(A)$ has closed range, $N\left(T_{\mathcal{T}, \mathcal{S}}(A)\right)=\mathcal{S} \cap N(A)$ and $N\left(T_{\mathcal{S}, \mathcal{T}}\left(A^{*}\right)\right)=\mathcal{T} \cap N\left(A^{*}\right)$.

Proof. Assume that $A$ is $(\mathcal{S}, \mathcal{T})$-complementable and $A(\mathcal{S}), A^{*}(\mathcal{T})$ are closed subspaces. Thus, $\mathcal{H}=\mathcal{S}+\left(A^{*} \mathcal{T}\right)^{\perp}$ and $\mathcal{K}=\mathcal{T}+(A \mathcal{S})^{\perp}$ or, equivalently, $\mathcal{S}^{\perp} \dot{+} A^{*} \mathcal{T}$ and $\mathcal{T}^{\perp} \dot{+} A \mathcal{S}$ are closed subspaces. Hence, by Proposition 3.4 and Lemma 3.3 we obtain item 2.

Conversely, suppose that item 2 holds. Then as $T_{\mathcal{T}, \mathcal{S}}(A)$ has closed range and $N\left(T_{\mathcal{T}, \mathcal{S}}(A)\right)=$ $\mathcal{S} \cap N(A)$ we get that $A(\mathcal{S}) \dot{+} \mathcal{T}^{\perp}$ is closed. Then $A \mathcal{S}$ is closed and $\mathcal{H}=\mathcal{T}+(A \mathcal{S})^{\perp}$. Similarly, but considering that $T_{\mathcal{S}, \mathcal{T}}\left(A^{*}\right)$ has also closed range and $N\left(T_{\mathcal{S}, \mathcal{T}}\left(A^{*}\right)\right)=\mathcal{T} \cap N\left(A^{*}\right)$, we get that $A^{*} \mathcal{T}$ is closed and $\mathcal{K}=\mathcal{S}+\left(A^{*} \mathcal{T}\right)^{\perp}$. Therefore, $A$ is $(\mathcal{S}, \mathcal{T})$-complementable.

We devote the remain of this section to study the relationship between the invertibility of $T_{\mathcal{T}, \mathcal{S}}(A)$ and the notion of complementability. For that, in the following result we collect some equivalent conditions to the invertibility of $T_{\mathcal{T}, \mathcal{S}}(A)$.

Proposition 3.7. The next conditions are equivalent:

1. $T_{\mathcal{T}, \mathcal{S}}(A)$ is invertible

2. $\mathcal{H}=A(\mathcal{S}) \dot{+} \mathcal{T}^{\perp}$ and $N(A) \cap \mathcal{S}=\{0\}$.

3. $\mathcal{H}=(A \mathcal{S})^{\perp} \dot{+} \mathcal{T}, N(A) \cap \mathcal{S}=\{0\}$ and $A(\mathcal{S})$ is closed.

Proof. $1 \Rightarrow 2$. Clearly, if $T_{\mathcal{T}, \mathcal{S}}(A)$ is invertible then $R\left(P_{\mathcal{T}} A P_{\mathcal{S}}\right)=\mathcal{T}$. Thus, $\mathcal{H}=P_{\mathcal{T}}^{-1}\left(R\left(P_{\mathcal{T}} A P_{\mathcal{S}}\right)\right)=$ $P_{\mathcal{T}}^{-1}\left(P_{\mathcal{T}}(A(\mathcal{S}))\right)=A(\mathcal{S})+\mathcal{T}^{\perp}$. Then, the result follows by Corollary 3.5.

$2 \Rightarrow 1$. If $\mathcal{H}=A(\mathcal{S}) \dot{+} \mathcal{T}^{\perp}$ then $\mathcal{T}=P_{\mathcal{T}}(\mathcal{H})=P_{\mathcal{T}}(A(\mathcal{S}))=R\left(P_{\mathcal{T}} A P_{\mathcal{S}}\right)$, i.e., $T_{\mathcal{T}, \mathcal{S}}(A)$ is surjective. The proof is complete by considering Corollary 3.5.

The equivalence $3 \Leftrightarrow 1$ follows from the fact that $\mathcal{H}=A \mathcal{S}+\mathcal{T}^{\perp}$ if and only if $\mathcal{H}=(A \mathcal{S})^{\perp} \dot{+} \mathcal{T}$ and $A \mathcal{S}$ is closed, see [14, Theorem 15]. 
We refer the reader to [15, Theorem 1 and Lemma 3] and [21, Theorems 1 and 2] for some other equivalent conditions to the invertibility of $T_{\mathcal{T}, \mathcal{S}}(A)$.

Corollary 3.8. $T_{\mathcal{T}, \mathcal{S}}(A)$ is invertible if and only if $T_{\mathcal{S}, \mathcal{T}}\left(A^{*}\right)$ is invertible.

Proof. By Proposition 3.7, $T_{\mathcal{T}, \mathcal{S}}(A)$ is invertible if and only if $R\left(P_{\mathcal{T}} A P_{\mathcal{S}}\right)=R\left(P_{\mathcal{T}}\right)$ and $N\left(P_{\mathcal{T}} A P_{\mathcal{S}}\right)=$ $N\left(P_{\mathcal{S}}\right)$ or, equivalently, $N\left(P_{\mathcal{S}} A^{*} P_{\mathcal{T}}\right)=N\left(P_{\mathcal{T}}\right)$ and $R\left(P_{\mathcal{S}} A^{*} P_{\mathcal{T}}\right)=R\left(P_{\mathcal{S}}\right)$, i.e., applying again Proposition $3.7, T_{\mathcal{S}, \mathcal{T}}\left(A^{*}\right)$ is invertible.

Corollary 3.9. If $T_{\mathcal{T}, \mathcal{S}}(A)$ is invertible then $A$ is $(\mathcal{S}, \mathcal{T})$-complementable.

Proof. Assume that $T_{\mathcal{T}, \mathcal{S}}(A)$ is invertible. Then, by Proposition 3.7, $N(A) \cap \mathcal{S}=N\left(T_{\mathcal{T}, \mathcal{S}}(A)\right)=\{0\}$ and $N\left(T_{\mathcal{S}, \mathcal{T}}\left(A^{*}\right)\right)=\mathcal{T} \cap N\left(A^{*}\right)=\{0\}$. Then, by Theorem 3.6, $A$ is $(\mathcal{S}, \mathcal{T})$-complementable.

Theorem 3.10. The next conditions are equivalent:

1. $T_{\mathcal{T}, \mathcal{S}}(A)$ is invertible.

2. $\mathcal{P}(A, \mathcal{S}, \mathcal{T})$ has a unique element and $A(\mathcal{S})$ is closed.

Moreover, if the above conditions hold then

$$
T_{\mathcal{T}, \mathcal{S}}(A)^{-1}=\left.(A P)^{\dagger} Q_{A \mathcal{S} / / \mathcal{T}}\right|_{\mathcal{T}}
$$

Proof. $1 \Rightarrow 2$. Suppose that $T_{\mathcal{T}, \mathcal{S}}(A)$ is invertible. By Corollary $3.9, A$ is $(\mathcal{S}, \mathcal{T})$-complementable or, equivalently, $\mathcal{P}(A, \mathcal{S}, \mathcal{T})$ is not empty. Moreover, by Proposition 3.7, $A \mathcal{S}$ is closed and $\left(A^{*}\right)^{-1}\left(\mathcal{S}^{\perp}\right) \cap$ $\mathcal{T}=\{0\}$. Applying again Proposition 3.7 to $T_{\mathcal{S}, \mathcal{T}}\left(A^{*}\right)$ (which is also invertible because of Corollary 3.8) we obtain that $A^{-1}\left(\mathcal{T}^{\perp}\right) \cap \mathcal{S}=\{0\}$. Therefore, by Corollary $2.12, \mathcal{P}(A, \mathcal{S}, \mathcal{T})$ has a unique element.

$2 \Rightarrow 1$. Suppose that $\mathcal{P}(A, \mathcal{S}, \mathcal{T})$ has a unique element and $A \mathcal{S}$ is closed. Then, by Corollary 2.12, $\mathcal{H}=\left(A^{*}\right)^{-1}\left(\mathcal{S}^{\perp}\right) \dot{+} \mathcal{T}$ and $\mathcal{S} \cap A^{-1}\left(\mathcal{T}^{\perp}\right)=\{0\}$. Now, $N(A) \cap \mathcal{S} \subseteq \mathcal{S} \cap A^{-1}\left(\mathcal{T}^{\perp}\right)$, i.e., $N(A) \cap \mathcal{S}=\{0\}$. Therefore, by Proposition 3.7, $T_{\mathcal{T}, \mathcal{S}}(A)$ is invertible. Finally, formula (7) follows by Theorem 3.2, noticing that if $\mathcal{P}(A, \mathcal{S}, \mathcal{T})=\{(E, F)\}$ then $F=Q_{\mathcal{T} / /(A \mathcal{S})^{\perp}}$ or, equivalently, $Q_{A \mathcal{S} / / \mathcal{T}^{\perp}}=F^{*}$.

\section{Bilateral shorted operator}

As mentioned in the introduction, the bilateral shorted operator defined in [4] extends to Hilbert spaces the matricial analogue introduced by Mitra and Puri [26] and Carlson and Haynsworth [9]. Next, we recall the definition given in [4], where we have interchanged $\mathcal{S}, \mathcal{T}$ and $\mathcal{S}^{\perp}, \mathcal{T}^{\perp}$ to maintain notational consistency with the previous concepts. In what follows, we consider the matrix form of an operator $A \in L(\mathcal{H}, \mathcal{K})$ given in (2).

Definition 4.1. If $A$ is $(\mathcal{S}, \mathcal{T})$-weakly complementable, the bilateral shorted operator of $A$ to the subspaces $\mathcal{S}, \mathcal{T}$ is the operator

$$
A_{/ \mathcal{S}, \mathcal{T}}:=\left(\begin{array}{cc}
0 & 0 \\
0 & A_{22}-C^{*} U^{*} B
\end{array}\right)
$$

where $B=\left(\left|A_{11}^{*}\right|^{1 / 2}\right)^{\dagger} A_{12}, C=\left(\left|A_{11}\right|^{1 / 2}\right)^{\dagger} A_{21}^{*}$, and $A_{11}^{*}=U^{*}\left|A_{11}^{*}\right|$ is the polar decomposition of $A_{11}^{*}$. 
The aim of this section is to provide new characterizations of $A_{/ \mathcal{S}, \mathcal{T}}$ by means of the densely defined projections given by the weakly-complementability of $A$. In this direction, our first result is a formula for $A_{/ \mathcal{S}, \mathcal{T}}$ in terms of the semiclosed projections $E_{0}, F_{0}$ given by (4) and (5):

Theorem 4.2. If $A$ is $(\mathcal{S}, \mathcal{T})$-weakly complementable then,

$$
A_{/ \mathcal{S}, \mathcal{T}}=\left(I-F_{0}\right) A \text { and } A_{/ \mathcal{T}, \mathcal{S}}^{*}=\left(I-E_{0}\right) A^{*},
$$

where $E_{0}, F_{0}$ are the densely defined projections given by (4) and (5), respectively.

Proof. Let $A$ be $(\mathcal{S}, \mathcal{T})$-weakly complementable. Then, by Remark 2.16,

$$
A=\underset{\mathcal{T}^{\perp}}{\mathcal{T}}\left(\begin{array}{cc}
\left|A_{11}^{*}\right| U & \left|A_{11}^{*}\right|^{1 / 2} B \\
C^{*}\left|A_{11}\right|^{1 / 2} & A_{22}
\end{array}\right) \stackrel{\mathcal{S}}{\mathcal{S}}
$$

where $A_{11}^{*}=U^{*}\left|A_{11}^{*}\right|$ is the polar decomposition of $A_{11}^{*}, B=\left(\left|A_{11}^{*}\right|^{1 / 2}\right)^{\dagger} A_{12}$, and $C=\left(\left|A_{11}\right|^{1 / 2}\right)^{\dagger} A_{21}^{*}$. Then, a simple computation shows that $\left(I-F_{0}\right) A=\left(\begin{array}{cc}0 & 0 \\ 0 & A_{22}-C^{*} U^{*} B\end{array}\right)=A_{/ \mathcal{S}, \mathcal{T}}$ and $\left(I-E_{0}\right) A^{*}=$ $\left(\begin{array}{cc}0 & 0 \\ 0 & A_{22}^{*}-B^{*} U C\end{array}\right)=A_{/ \mathcal{T}, \mathcal{S}}^{*}$, where $E_{0}$ and $F_{0}$ are given by (4) and (5), respectively.

For a positive operator $A \in L(\mathcal{H})$, Krein [23] proved that the shorted operator $A_{/ \mathcal{S}}$ turns out to be the maximum of all positive operators which are smaller than $A$ with respect to the Lowner order (i.e., $A \leq B$ if $\langle A x, x\rangle \leq\langle B x, x\rangle \forall x \in \mathcal{H}$ ) and which have range lying in the fixed subspace $\mathcal{S}^{\perp}$. See also Anderson and Trapp [2], Pekarev-Šmuljan [28] and Arlinskii and E. Tsekanovskii [5]. For $A \in L(\mathcal{H})$ non positive, Mitra [25] proved (for matrices in $\mathbb{C}^{m \times n}$ ) that a similar result can be obtained if the minus order is considered. More precisely, he proved that

$$
A_{/ \mathcal{s}, \mathcal{T}}=\max _{\bar{\Sigma}}\left\{C \in \mathbb{C}^{m \times n}: C \overline{\leq} A, R(C) \subseteq \mathcal{T}^{\perp} \text { and } R\left(C^{*}\right) \subseteq \mathcal{S}^{\perp}\right\}
$$

where $A \overline{\leq} B$ if $R(A) \cap R(B-A)=\{0\}$ and $R\left(A^{*}\right) \cap R\left(A^{*}-B^{*}\right)=\{0\}$. Later, in order to extend this result for bounded operators on infinite dimensional Hilbert spaces, Antezana et al. [4] introduced the next generalization of the minus order: given $A, B \in L(\mathcal{H}, \mathcal{K}), A \overline{\leq} B$ if $\overline{R(A)} \dot{+} \overline{R(B-A)}$ and $\overline{R\left(A^{*}\right)}+\overline{R\left(B^{*}-A^{*}\right)}$ are closed subspaces, or equivalently, there are bounded (oblique) projections $P$ and $Q$ such that $A=P B$ and $A^{*}=Q B^{*}$, see [4, Proposition 4.13]. See also [16], where it was proved that $A \overline{\leq} B$ if and only if $R(B)=R(A)+R(B-A)$ and $R\left(B^{*}\right)=R\left(A^{*}\right)+R\left(B^{*}-A^{*}\right)$. If $A$ is $(\mathcal{S}, \mathcal{T})$-complementable then

$$
A_{/ \mathcal{S}, \mathcal{T}}:=\max _{\overline{\underline{x}}}\left\{C \in L(\mathcal{H}, \mathcal{K}): C \overline{\leq} A, R(C) \subseteq \mathcal{T}^{\perp}, R\left(C^{*}\right) \subseteq \mathcal{S}^{\perp}\right\},
$$

see [4, Theorem 4.15]. This result extends Mitra's result under the hypothesis of complementability.

Our goal now is to extend, under the condition of weakly-complementability, the variational characterization (10) of the bilateral shorted operator. For this, we define a partial order in $L(\mathcal{H}, \mathcal{K})$ that extends the minus order:

Definition 4.3. Given two operators $A, B \in L(\mathcal{H}, \mathcal{K})$, we shall write $A \prec B$ if there exist two densely defined projections $Q, P$ with closed ranges such that $A=Q B$ and $A^{*}=P B^{*}$.

Lemma 4.4. Let $A, B \in L(\mathcal{H}, \mathcal{K})$. The next conditions are equivalent: 
1. $A \prec B$.

2. $\overline{R(A)} \cap R(B-A)=\{0\}$ and $\overline{R\left(A^{*}\right)} \cap R\left(B^{*}-A^{*}\right)=\{0\}$.

Proof. Assume that $A \prec B$, and let $Q, P$ be two densely defined projections with closed ranges such that $A=Q B$ and $A^{*}=P B^{*}$. Without loss of generality, we can assume that $R(Q)=\overline{R(A)}$ and $R(P)=\overline{R\left(A^{*}\right)}$. Hence, $Q(B-A)=0$, i.e., $R(B-A) \subseteq N(Q)$ and so $\overline{R(A)} \cap R(B-A)=$ $\{0\}$. Similarly, $\overline{R\left(A^{*}\right)} \cap R\left(B^{*}-A^{*}\right)=\{0\}$. Conversely, suppose that $\overline{R(A)} \cap R(B-A)=\{0\}$

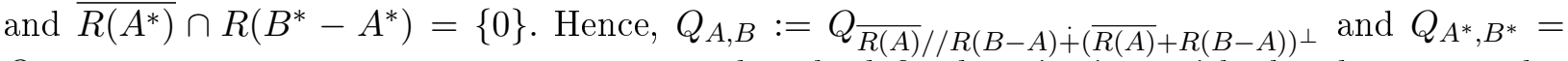
$Q \overline{R\left(A^{*}\right) / / R\left(B^{*}-A^{*}\right) \dot{+}\left(\overline{R\left(A^{*}\right)}+R\left(B^{*}-A^{*}\right)\right)^{\perp}}$ are two densely defined projections with closed ranges and a simple computation shows that $A=Q_{A, B} B$ and $A^{*}=Q_{A^{*}, B^{*}} B^{*}$. Therefore, $A \prec B$.

From now on, given $A, B \in L(\mathcal{H}, \mathcal{K})$ we denote by

$$
\prec_{A, B}:=\{Q: Q \text { is a densely defined projection with closed range s.t. } A=Q B\} \text {. }
$$

Thus, $A \prec B$ if and only if $\prec_{A, B} \neq \emptyset$ and $\prec_{A^{*}, B^{*}} \neq \emptyset$. Moreover, if $A, B \in L(\mathcal{H}, \mathcal{K})$ verify that

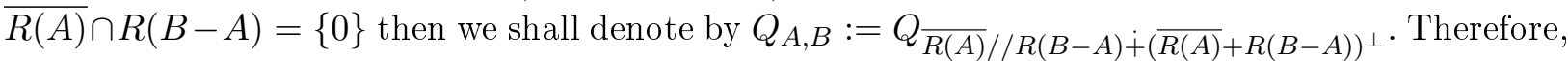
taking into account the proof of Lemma 4.4, if $A \prec B$ then $Q_{A, B} \in \prec A, B$ and $Q_{A^{*}, B^{*}} \in \prec A^{*}, B^{*}$.

Let us prove that $\prec$ defines an order in $L(\mathcal{H}, \mathcal{K})$ :

Lemma 4.5. The relation $\prec$ is a partial order in $L(\mathcal{H}, \mathcal{K})$.

Proof. In fact, the relation $\prec$ is:

1. Reflexive: for all $A \in L(\mathcal{H}, \mathcal{K}), A=P_{\overline{R(A)}} A$, and $A^{*}=P_{\overline{R\left(A^{*}\right)}} A^{*}$ i.e., $A \prec A$.

2. Antisymmetric: If $A \prec B$ and $B \prec A$ then $A^{*}=Q_{A^{*}, B^{*}} B^{*}$, so $N\left(B^{*}\right) \subseteq N\left(A^{*}\right)$, and $B^{*}=Q_{B^{*}, A^{*}} A^{*}$, so $N\left(A^{*}\right) \subseteq N\left(B^{*}\right)$. Thus, $N\left(A^{*}\right)=N\left(B^{*}\right)$ or, equivalently, $\overline{R(A)}=\overline{R(B)}$. Hence, $A=Q_{A, B} B=B$.

3. Transitive: Let $A \prec B$ and $B \prec C$. Then, $A=Q_{A B} B=Q_{A, B} Q_{B, C} C$. Let us prove that $Q_{A, B} Q_{B, C}$ is a densely defined projection with closed range. In fact, $N\left(Q_{B, C}\right) \subseteq$ $\mathcal{D}\left(Q_{A, B} Q_{B, C}\right)$ and $R(B) \subseteq \mathcal{D}\left(Q_{A, B} Q_{B, C}\right)$. Thus $N\left(Q_{B, C}\right)+R(B) \subseteq \mathcal{D}\left(Q_{A, B} Q_{B, C}\right)$, and so $Q_{A, B} Q_{B, C}$ is densely defined. To see that $Q_{A, B} Q_{B, C}$ is a projection, $R\left(Q_{A, B} Q_{B, C}\right) \subseteq$ $R\left(Q_{A, B}\right)=\overline{R(A)} \subseteq \overline{R(B)} \subseteq \mathcal{D}\left(Q_{B, C}\right)$. Hence, $Q_{B, C} Q_{A, B} Q_{B, C}=Q_{A, B} Q_{B, C}$ in $\mathcal{D}\left(Q_{A, B} Q_{B, C}\right)$ and so $Q_{A, B} Q_{B, C}=\left(Q_{A, B} Q_{B, C}\right)^{2}$. Finally, $R\left(Q_{A, B} Q_{B, C}\right) \subseteq \overline{R(A)}$. On the other hand, as $\overline{R(A)} \subseteq \overline{R(B)}$, then $Q_{B, C} Q_{A, B}=Q_{A, B}$. Thus, $Q_{A, B} Q_{B, C} Q_{A, B}=Q_{A, B}$ and so $\overline{R(A)}=$ $R\left(Q_{A, B}\right) \subseteq R\left(Q_{A, B} Q_{B, C}\right)$. Therefore, $R\left(Q_{A, B} Q_{B, C}\right)=\overline{R(A)}$ is closed.

Similarly, $A^{*}=Q_{A^{*}, B^{*}} B^{*}=Q_{A^{*}, B^{*}} Q_{B^{*}, C^{*}} C^{*}$ and $Q_{A^{*}, B^{*}} Q_{B^{*}, C^{*}}$ is a densely defined projection with range $\overline{R\left(A^{*}\right)}$. Then, $A \prec C$.

Clearly, the partial order $\prec$ extends the minus order, in the sense that if $A \leq B$ then $A \prec B$.

Now we are in position to prove the last result of this paper. If $A$ is $(\mathcal{S}, \mathcal{T})$-weakly complementable, consider the factorization given in (3) and let $\Gamma_{1}:=\left(\begin{array}{cc}\left|A_{11}^{*}\right|^{1 / 2} & 0 \\ 0 & I\end{array}\right)$ and $\Gamma_{2}:=$ $\left(\begin{array}{cc}\left|A_{11}\right|^{1 / 2} & 0 \\ 0 & I\end{array}\right)$. Notice that $A=\Gamma_{1} A_{0} \Gamma_{2}$ where $A_{0}=\left(\begin{array}{cc}U & B \\ C^{*} & A_{22}\end{array}\right)$. Hence, define

$\mathcal{M}^{\prec}(A, \mathcal{S}, \mathcal{T})=\left\{C \in L(\mathcal{H}, \mathcal{K}): C \prec A, R(C) \subseteq \mathcal{T}^{\perp}, R\left(C^{*}\right) \subseteq \mathcal{S}^{\perp}, \exists Q \in \prec_{C, A}, \exists P \in \prec_{C^{*}, A^{*}}\right.$ s.t. $\left.Q \Gamma_{1} \in L(\mathcal{K}), P \Gamma_{2} \in L(\mathcal{H})\right\}$. 
Theorem 4.6. Let $A$ be $(\mathcal{S}, \mathcal{T})$-weakly complementable. Then,

$$
A_{/ \mathcal{S}, \mathcal{T}}=\max _{\prec} \mathcal{M}^{\prec}(A, \mathcal{S}, \mathcal{T}) \text {. }
$$

Proof. Let us start by showing that $A_{/ \mathcal{S}, \mathcal{T}} \in \mathcal{M}^{\prec}(A, \mathcal{S}, \mathcal{T})$. By Theorem $4.2, A_{/ \mathcal{S}, \mathcal{T}}=\left(I-F_{0}\right) A$ and $\left(A_{/ \mathcal{S}, \mathcal{T}}\right)^{*}=\left(I-E_{0}\right) A^{*}$, where $E_{0}, F_{0}$ are given by (4) and (5), respectively. Thus, since $I-E_{0}$ and $I-F_{0}$ are two densely defined projections with closed ranges $\left(R\left(I-F_{0}\right)=N\left(F_{0}\right)=\mathcal{T}^{\perp}\right.$ and $\left.R\left(I-E_{0}\right)=N\left(E_{0}\right)=\mathcal{S}^{\perp}\right)$, we get that $A_{/ \mathcal{S}, \mathcal{T}} \prec A, R\left(A_{/ \mathcal{S}, \mathcal{T}}\right) \subseteq \mathcal{T}^{\perp}$ and $R\left(A_{/ \mathcal{S}, \mathcal{T}}^{*}\right) \subseteq \mathcal{S}^{\perp}$. Moreover, $\left(I-F_{0}\right) \Gamma_{1}=\left(I-F_{0}\right)\left(\left|P_{\mathcal{S}} A^{*} P_{\mathcal{T}}\right|^{1 / 2}+P_{\mathcal{T}^{\perp}}\right) \in L(\mathcal{K})$ and $\left(I-E_{0}\right) \Gamma_{2}=\left(I-E_{0}\right)\left(\left|P_{\mathcal{T}} A P_{\mathcal{S}}\right|^{1 / 2}+P_{\mathcal{S}^{\perp}}\right) \in$ $L(\mathcal{H})$ because $E_{0}$ and $F_{0}$ verify the conditions of Definition 2.14. Then, $A_{/ \mathcal{S}, \mathcal{T}} \in \mathcal{M}^{\prec}(A, \mathcal{S}, \mathcal{T})$.

Now, consider $C \in \mathcal{M}^{\prec}(A, \mathcal{S}, \mathcal{T})$. Then, $C=Q A=Q \Gamma_{1} A_{0} \Gamma_{2}$. Therefore, as $Q \Gamma_{1} \in L(\mathcal{K})$, we have that $C^{*}=\Gamma_{2} A_{0}^{*}\left(Q \Gamma_{1}\right)^{*}=\left(I-E_{0}\right) \Gamma_{2} A_{0}^{*}\left(Q \Gamma_{1}\right)^{*}$, because $R\left(C^{*}\right) \subseteq \mathcal{S}^{\perp}=R\left(I-E_{0}\right)$. Moreover, $\left(I-E_{0}\right) \Gamma_{2} \in L(\mathcal{H})$. Hence, $C=Q \Gamma_{1} A_{0}\left(\left(I-E_{0}\right) \Gamma_{2}\right)^{*}$. We claim that $A_{/ \mathcal{s}, \mathcal{T}}=\Gamma_{1} A_{0}\left(\left(I-E_{0}\right) \Gamma_{2}\right)^{*}$. In fact, $A_{/ \mathcal{S}, \mathcal{T}}=\left(\left(I-E_{0}\right) A^{*}\right)^{*}=\left(\left(I-E_{0}\right) \Gamma_{2} A_{0}^{*} \Gamma_{1}\right)^{*}=\Gamma_{1} A_{0}\left(\left(I-E_{0}\right) \Gamma_{2}\right)^{*}$, as desired. Then, $C=Q A / \mathcal{S}, \mathcal{T}$.

On the other hand, $C^{*}=P A^{*}=P \Gamma_{2} A_{0}^{*} \Gamma_{1}$. Hence, as $P \Gamma_{2} \in L(\mathcal{H})$, we get that $C=$ $\Gamma_{1} A_{0}\left(P \Gamma_{2}\right)^{*}=\left(I-F_{0}\right) \Gamma_{1} A_{0}\left(P \Gamma_{2}\right)^{*}$, because $R(C) \subseteq \mathcal{T}^{\perp}=R\left(I-F_{0}\right)$. Then, $C^{*}=P \Gamma_{2} A_{0}^{*}((I-$ $\left.\left.F_{0}\right) \Gamma_{1}\right)^{*}$. Now, $\left(A_{/ \mathcal{S}, \mathcal{T}}\right)^{*}=\Gamma_{2} A_{0}^{*}\left(\left(I-F_{0}\right) \Gamma_{1}\right)^{*}$ because $A_{/ \mathcal{S}, \mathcal{T}}=\left(I-F_{0}\right) A=\left(I-F_{0}\right) \Gamma_{1} A_{0} \Gamma_{2}$ and $\left(I-F_{0}\right) \Gamma_{1} \in L(\mathcal{K})$. Summarizing, $C=Q A_{/ \mathcal{S}, \mathcal{T}}$ and $C^{*}=P\left(A_{/ \mathcal{S}, \mathcal{T}}\right)^{*}$, i.e., $C \prec A_{/ \mathcal{s}, \mathcal{T}}$. The proof is complete.

\section{References}

[1] W. N. Anderson, Jr. and R. J. Duffin, Series and parallel addition of matrices, J. Math. Anal. Appl. 26 (1969), 576-594.

[2] W. N. Anderson, Jr. and G. E. Trapp, Shorted operators. II, SIAM J. Appl. Math. 28 (1975), $60-71$.

[3] Tsuyoshi Ando, Generalized Schur complements, Linear Algebra Appl. 27 (1979), 173-186.

[4] Jorge Antezana, Gustavo Corach, and Demetrio Stojanoff, Bilateral shorted operators and parallel sums, Linear Algebra Appl. 414 (2006), no. 2-3, 570-588.

[5] Yu. Arlinskiǔ and E. Tsekanovskiŭ, M. Kreı̆n's research on semi-bounded operators, its contemporary developments, and applications, Modern analysis and applications. The Mark Krein Centenary Conference. Vol. 1: Operator theory and related topics, Oper. Theory Adv. Appl., vol. 190, Birkhäuser Verlag, Basel, 2009, pp. 65-112.

[6] Don Buckholtz, Hilbert space idempotents and involutions, Proc. Amer. Math. Soc. 128 (2000), no. $5,1415-1418$.

[7] Charles A. Butler and Thomas D. Morley, Six generalized Schur complements, Linear Algebra Appl. 106 (1988), 259-269.

[8] David Carlson, What are Schur complements, anyway?, Linear Algebra Appl. 74 (1986), 257275.

[9] David Carlson and Emilie V. Haynsworth, Complementable and almost definite matrices, Linear Algebra Appl. 52/53 (1983), 157-176. 
[10] Gustavo Corach, Alejandra Maestripieri, and Demetrio Stojanoff, Oblique projections and Schur complements, Acta Sci. Math. (Szeged) 67 (2001), no. 1-2, 337-356.

[11] Gustavo Corach, Alejandra Maestripieri, and Demetrio Stojanoff, Generalized Schur complements and oblique projections, Linear Algebra Appl. 341 (2002), 259-272, Special issue dedicated to Professor T. Ando.

[12] Gustavo Corach, Alejandra Maestripieri, and Demetrio Stojanoff, A classification of projectors, Topological algebras, their applications, and related topics, Banach Center Publ., vol. 67, Polish Acad. Sci. Inst. Math., Warsaw, 2005, pp. 145-160.

[13] Richard W. Cottle, Manifestations of the Schur complement, Linear Algebra and Appl. 8 (1974), 189-211.

[14] Frank Deutsch, The angle between subspaces of a Hilbert space, Approximation theory, wavelets and applications (Maratea, 1994), NATO Adv. Sci. Inst. Ser. C Math. Phys. Sci., vol. 454, Kluwer Acad. Publ., Dordrecht, 1995, pp. 107-130.

[15] Allen Devinatz and Marvin Shinbrot, General Wiener-Hopf operators, Trans. Amer. Math. Soc. 145 (1969), 467-494.

[16] Marko S. Djikić, Guillermina Fongi, and Alejandra Maestripieri, The minus order and range additivity, Linear Algebra Appl. 531 (2017), 234-256.

[17] Ronald G. Douglas, On majorization, factorization, and range inclusion of operators on Hilbert space, Proc. Amer. Math. Soc. 17 (1966), 413-415.

[18] P. A. Fillmore and J. P. Williams, On operator ranges, Advances in Math. 7 (1971), 254-281.

[19] Seppo Hassi and Kenneth Nordström, On projections in a space with an indefinite metric, Linear Algebra Appl. 208/209 (1994), 401-417.

[20] Emilie V. Haynsworth, Determination of the inertia of a partitioned Hermitian matrix, Linear Algebra and Appl. 1 (1968), no. 1, 73-81.

[21] James R. Holub, On invertibility of Wiener-Hopf operators, Math. Japon. 25 (1980), no. 3, $341-343$.

[22] William E. Kaufman, Semiclosed operators in Hilbert space, Proc. Amer. Math. Soc. 76 (1979), no. $1,67-73$.

[23] Mark G. Kreı̆n, The theory of self-adjoint extensions of semi-bounded Hermitian transformations and its applications. II, Mat. Sbornik N.S. 21(63) (1947), 365-404.

[24] Michael Lauzon and Sergei Treil, Common complements of two subspaces of a Hilbert space, J. Funct. Anal. 212 (2004), no. 2, 500-512.

[25] Sujit Kumar Mitra, The minus partial order and the shorted matrix, Linear Algebra Appl. 83 (1986), 1-27.

[26] Sujit Kumar Mitra and Madan L. Puri, Shorted matrices-an extended concept and some applications, Linear Algebra Appl. 42 (1982), 57-79.

[27] Schōichi Ōta, Unbounded nilpotents and idempotents, J. Math. Anal. Appl. 132 (1988), no. 1, 300-308. 
[28] È. L. Pekarev and Ju. L. Šmul jan, Parallel addition and parallel subtraction of operators, Izv. Akad. Nauk SSSR Ser. Mat. 40 (1976), no. 2, 366-387, 470.

[29] Victor J. Pellegrini, General Wiener-Hopf operators and the numerical range of an operator, Proc. Amer. Math. Soc. 38 (1973), 141-146.

[30] Issai Schur, Über Potenzreihen, die im Innern des Einheitskreises beschränkt sind, J. Reine Angew. Math. 147 (1917), 205-232.

[31] Marvin Shinbrot, On singular integral operations, J. Math. Mech. 13 (1964), 395-406.

[32] Angus E. Taylor and David C. Lay, Introduction to functional analysis, second ed., Robert E. Krieger Publishing Co., Inc., Melbourne, FL, 1986.

[33] Fuzhen Zhang (ed.), The Schur complement and its applications, Numerical Methods and Algorithms, vol. 4, Springer-Verlag, New York, 2005.

1 Instituto Argentino de Matemática "Alberto P. Calderón", CONICET, Buenos Aires, Argentina

${ }^{2}$ Universidad de Buenos Aires, Facultad de Ingeniería, Departamento de Matemática, Buenos Aires, Argentina.

${ }^{a}$ lauraarias@conicet.gov.ar, ${ }^{b}$ gcorach@fi.uba.ar, ${ }^{c}$ amaestri@fi.uba.ar 